QUARTERLY OF APPLIED MATHEMATICS

VOLUME LXIV, NUMBER 1

MARCH 2006, PAGES 167-188

S $0033-569 \mathrm{X}(06) 01004-0$

Article electronically published on January 24, 2006

\title{
CONVERGENCE TO EQUILIBRIUM FOR THE DAMPED SEMILINEAR WAVE EQUATION WITH CRITICAL EXPONENT AND DISSIPATIVE BOUNDARY CONDITION
}

BY

HAO WU (Institute of Mathematics, Fudan University, 200433 Shanghai, P.R. China)

AND

SONGMU ZHENG (Institute of Mathematics, Fudan University, 200433 Shanghai, P.R. China)

Abstract. This paper is concerned with the asymptotic behavior of the solution to the following damped semilinear wave equation with critical exponent:

$$
u_{t t}+u_{t}-\Delta u+f(x, u)=0, \quad(x, t) \in \Omega \times \mathbb{R}^{+}
$$

subject to the dissipative boundary condition

$$
\partial_{\nu} u+u+u_{t}=0, \quad t>0, x \in \Gamma
$$

and the initial conditions

$$
\left.u\right|_{t=0}=u_{0}(x),\left.\quad u_{t}\right|_{t=0}=u_{1}(x), \quad x \in \Omega,
$$

where $\Omega$ is a bounded domain in $\mathbb{R}^{3}$ with smooth boundary $\Gamma, \nu$ is the outward normal direction to the boundary, and $f$ is analytic in $u$. In this paper convergence of the solution to an equilibrium as time goes to infinity is proved. While these types of results are known for the damped semilinear wave equation with interior dissipation and Dirichlet boundary condition, this is, to our knowledge, the first result with dissipative boundary condition and critical growth exponent.

1. Introduction. In this paper we consider the following damped semilinear wave equation with critical exponent:

$$
u_{t t}+u_{t}-\Delta u+f(x, u)=0, \quad(x, t) \in \Omega \times \mathbb{R}^{+}
$$

Received July 18, 2005.

2000 Mathematics Subject Classification. Primary 35B40, 35Q99.

Key words and phrases. Semilinear wave equation, critical growth exponent, dissipative boundary condition, Simon-Lojasiewciz inequality.

The authors are supported by the NSF of China under grant No. 10371022 and by the Ministry of Education in China under grant No. 20050246002, and by Key Laboratory of Mathematics for Nonlinear Sciences in Fudan University sponsored by the Ministry of Education in China.

E-mail address: haowufd@yahoo.com

E-mail address: songmuzheng@yahoo.com 
subject to the dissipative boundary condition

$$
\partial_{\nu} u+u+u_{t}=0, \quad t>0, x \in \Gamma
$$

and the initial conditions

$$
\left.u\right|_{t=0}=u_{0}(x),\left.\quad u_{t}\right|_{t=0}=u_{1}(x), \quad x \in \Omega .
$$

In the above, $\Omega$ is a bounded domain in $\mathbb{R}^{3}$ with smooth boundary $\Gamma, \nu$ is the outward normal direction to the boundary, and $f$ is a nonlinear function satisfying the following assumptions.

(F1) $f$ is analytic in $u \in \mathbb{R}$, and $f$ is $C^{2}$ in $x \in \bar{\Omega}$. Furthermore,

$$
\left|f^{\prime \prime}(\cdot, s)\right| \leq c(1+|s|) \quad \forall s \in \mathbb{R}, \quad x \in \bar{\Omega} .
$$

Hereafter, we denote by $f^{\prime}(x, u), f^{\prime \prime}(x, u)$ the first-order derivative and the second-order derivative of $f$ with respect to $u$, respectively.

(F2) For all $x \in \bar{\Omega}$,

$$
\liminf _{|s| \rightarrow \infty} \frac{f(\cdot, s)}{s}>-\lambda,
$$

where $\lambda>0$ is the best Sobolev constant in the following imbedding inequality:

$$
\int_{\Omega}|\nabla u|^{2} d x+\int_{\Gamma} u^{2} d S \geq \lambda \int_{\Omega} u^{2} d x .
$$

Notice that the assumption (F1) implies that $f$ is allowed to grow cubically in $u$, i.e., the growth exponent of $f$ is critical in the case $n=3$. The mechanical meaning of boundary condition (1.2) simply implies that there is friction damping on the boundary $\Gamma$ which is linearly proportional to the velocity $u_{t}$.

By $H^{m}(\Omega)$ we denote the Sobolev spaces $W^{m, 2}(\Omega)$ with norm $\|\cdot\|_{H^{m}}$, and we simply denote the norm in $L^{2}(\Omega)$ by $\|\cdot\|$, and especially we equip $H^{1}(\Omega)$ with the norm

$$
\|u\|_{H^{1}(\Omega)}=\left(\int_{\Omega}|\nabla u|^{2} d x+\int_{\Gamma} u^{2} d S\right)^{1 / 2} .
$$

We also introduce the space $\mathcal{H} \equiv H^{1}(\Omega) \times L^{2}(\Omega)$, which is often referred to as the finite energy space.

Denote

$$
\mathcal{D}=\left\{(u, v)^{T} \in H^{2}(\Omega) \times H^{1}(\Omega)\left|\partial_{\nu} u+u+v\right|_{\Gamma}=0\right\},
$$

which clearly is a closed subspace of $H^{2}(\Omega) \times H^{1}(\Omega)$.

The main result of this paper is stated as follows.

Theorem 1.1. Suppose that the assumptions (F1), (F2) are satisfied. Then for any initial data $\left(u_{0}, u_{1}\right)^{T} \in \mathcal{D}$, problem (1.1)-(1.3) admits a unique global solution

$$
\left(u, u_{t}\right)^{T} \in C([0,+\infty) ; \mathcal{D}) \cap C^{1}([0,+\infty) ; \mathcal{H}), \quad u_{t t} \in C\left([0,+\infty) ; L^{2}(\Omega)\right) .
$$

Moreover, $\left(u(x, t), u_{t}(x, t)\right)^{T}$ converges to an equilibrium $(\psi(x), 0)^{T}$ in the topology of $\mathcal{H}$ as time goes to infinity, i.e.,

$$
\lim _{t \rightarrow+\infty}\left(\|u(\cdot, t)-\psi\|_{H^{1}(\Omega)}+\left\|u_{t}\right\|\right)=0 .
$$


Here $\psi(x)$ is an equilibrium to problem (1.1)-(1.3), i.e., $\psi(x)$ is a classical solution to the following nonlinear elliptic boundary value problem:

$$
\left\{\begin{array}{l}
-\Delta \psi+f(x, \psi)=0, \quad x \in \Omega \\
\partial_{\nu} \psi+\psi=0, \quad x \in \Gamma
\end{array}\right.
$$

Before giving the detailed proof of Theorem 1.1, let us first recall some related results in the literature.

The study of the asymptotic behavior of solutions to nonlinear dissipative evolution equations has attracted a lot of interest of many mathematicians for a long period of time. It can be divided into two categories: in the first category, it is concerned with the asymptotic behavior of a global solution (or a single orbit) for any given initial datum, i.e., whether the global solution will converge to an equilibrium as time goes to infinity; in the second category, it is concerned with the asymptotic behavior of a family of global solutions (or orbits) for initial data starting from any bounded set in a certain Sobolev space, i.e., whether the family of global solutions will converge to a compact invariant set, i.e., a global attractor. For the study of the second category, we refer to the three books: [32] by Temam, [11] by Hale and [2] by Babin and Vishik. As far as the damped semilinear wave equation is concerned, in the books [11] and [2], the exponent of the nonlinear term $f$ is allowed to be critical, i.e., for $n=3$, the growth exponent of $f$ in $u$ is allowed to be cubic. In this direction, we also refer to the paper [8] by Feireisl where the interior damping is allowed to be nonlinear. In this direction, we also refer to a very recent paper [3] by Ball on the results for the nonlinear term $f$ being only continuous. In that paper, comprehensive references for the damped semilinear wave equations are also given. Notice that in all these papers, the solution always satisfies the Dirichlet boundary condition.

More closely related with the problem we studied here, we refer to a recent paper 7 by Chueshov, Eller and Lasiecka where the existence of a global attractor is proved for the semilinear wave equations (1.1) without interior damping, but with nonlinear dissipative boundary condition. More precisely, they proved that with certain dissipative boundary conditions and nonlinear term $f$ being allowed to have critical exponent, then there is a global attractor which is compact in the finite energy space $\mathcal{H} \equiv H^{1}(\Omega) \times L^{2}(\Omega)$ defined previously. Moreover, when $f$ is subcritical, then the global attractor is a closed bounded set in $\mathcal{D}$ defined in (1.4).

We should notice that the study of two categories is closely related in the following sense: if the number of solutions to the stationary problem (1.6) were finite or discrete, then (1.5) would follow immediately from the result in [7. (see Corollary 5.4 in [7]). However, for the higher space dimension case, whether the set of equilibria is finite or a discrete set is an open problem, and in general it is not true (see, e.g., 26, 27, and the related reference cited in [3]).

Concerning the study of the first category, in 1978, Matano 24] considered the initial boundary value problems for the nonlinear parabolic equation in one space dimension and proved that if the global solution is bounded for all time, then as time goes to infinity, the solution must converge to an equilibrium. In other words, the $\omega$-limit set consists of a single point. We also refer to the paper [35] by Zelenyak in this direction. Since 
then, many attempts have been made to extend this result to higher space dimensions. However, the situation in higher space dimensions is very different. Examples have been given in [26, 27] showing that for a nonlinear parabolic equation with nonanalytic nonlinear term $f$, the corresponding initial Dirichlet boundary value problem has a bounded solution whose $\omega$-limit set is diffeomorphic to the unit circle $S^{1}$. On the other hand, various assumptions have been made in the literature to assure that any bounded global solution will converge to an equilibrium. Among these attempts, Simon in [31] made a major advance by proving that for semilinear parabolic problems if the nonlinear term is analytic in an unknown function $u$, then convergence to an equilibrium holds. His idea relies on a generalization of the Lojasiewicz inequality for analytic functions defined in the finite-dimensional space $\mathbb{R}^{m}$ (see 22], 20, 21]). Since then, much work in this direction has been done; see, e.g., [15, [28, [1], 14], 6], [9, 34], 19], [36] and the references cited there for the results on nonlinear parabolic equations, where in 34 the problem with dissipative boundary conditions is studied; see also a very recent paper [38] by Zheng and Chipot for the nonlinear parabolic equations with nonlocal terms.

As far as applications of the Simon-Lojasiewicz approach to the damped semilinear wave equations, the situation is more delicate mainly due to the following two reasons:

First, the semilinear wave equations are hyperbolic equations, and it turns out that they do not have the smoothing property, as nonlinear parabolic equations do. The condition of precompactness of solutions for damped semilinear wave equations is expected, i.e., the solution can be divided into two parts with one part being uniformly convergent to zero, and the other part of the solution being relatively compact. However, as Haraux and Jendoubi pointed out in their paper [13, precompactness is nontrivial, which is not always easy to check in practice.

Secondly, the application of the Simon-Lojasiewicz approach to the damped semilinear wave equations is not as straightforward as for the single nonlinear parabolic equation, due to the appearance of the term $u_{t t}$ in the equation (1.1). It turns out that an auxiliary functional, which varies from problem to problem, has to be introduced. We refer to the paper [16] by Jendoubi for the first attempt in this direction in the literature. See also [19, 36] for the similar situation for the study of nonlinear phase-field equations with Neumann boundary conditions.

We refer to [16 by Jendoubi, 13 by Haraux and Jendoubi regarding the study of convergence to equilibrium for the damped semilinear wave equation with nonlinear $f$ being analytic in $u$. In [16, although convergence to an equilibrium for the damped semilinear equation follows from the unproved assumption (which is the exact wording used in [13] by Haraux and Jendoubi) that $\left\{u, u_{t}\right\}$ is precompact in $W^{2, p}(\Omega) \times W^{1, p}(\Omega)$ and $f$ is analytic in $u$, the advantage of that paper is that with the previously mentioned unproved assumption there is no further growth assumption on $f$. In the later paper [13, aimed at removing that unproved assumption, they made the growth assumption on $f$ to obtain the convergence to equilibrium. Notice that (see Theorem 1.2, p. 97 in [13]), the growth condition in their paper is subcritical when $n=3$. We should also notice that in all these papers, the solution always satisfies the Dirichlet boundary condition.

The new features of our problem (1.1)-(1.3) are the following. First, we treat the dissipative boundary condition (1.2), which is important from a mechanical point of view, 
and it turns out that for the corresponding elliptic operator, it yields a nonhomogeneous boundary condition. As a result, the Simon-Lojasiewicz type inequality we are going to derive is naturally different from the one with the usual homogeneous Dirichlet boundary condition. Another feature is that the growth assumption on $f$ is critical, which implies that one could not use the result by Webb [33] to obtain the precompactness. Finally, we give a rigorous proof of precompactness (see Lemma 2.2 in Section 2). It turns out that we have to overcome the corresponding mathematical difficulties due to these new features, and to extend the usual Simon-Lojasiewicz inequality in our proof. Notice that our result requires that the initial data be in $\mathcal{D}$ instead of $\mathcal{H}$. This is natural because we now treat the dissipative boundary condition.

This paper is organized as follows. In Section 2 we give a brief sketch of the proof for the global existence of the solution. A key result in that section is to prove a uniform a priori estimate of the solution (Lemma 2.2). Notice that this lemma has never been given in the literature. In the paper [7] by Chueshov, Eller and Lasiecka, a similar result was proved under the subcritical growth case (see Theorem 1.5 in that paper) and we now treat the critical growth case. In Section 3 of this paper we derive the extended Simon-Lojasiewicz inequality. In Section 4 we give the detailed proof of Theorem 1.1.

2. Global existence and uniqueness. For the sake of the reader, we first briefly recall the related global existence and uniqueness results mainly based on the paper [7] by Chueshov, Eller and Lasiecka. Let

$$
\frac{1}{2}\left\|\left(u, u_{t}\right)^{T}\right\|_{\mathcal{H}}^{2}=\frac{1}{2} \int_{\Omega}|\nabla u|^{2} d x+\frac{1}{2} \int_{\Gamma} u^{2} d S+\frac{1}{2} \int_{\Omega} u_{t}^{2} d x
$$

and define the energy function as follows:

$$
\mathcal{E}\left(u(t), u_{t}(t)\right)=\frac{1}{2}\left\|\left(u, u_{t}\right)^{T}\right\|_{\mathcal{H}}^{2}+\int_{\Omega} F(x, u(x, t)) d x,
$$

where $F(x, z)=\int_{0}^{z} f(x, s) d s$.

In what follows we shall use classical nonlinear semigroup theory (see, e.g., 4], [5, [29], 37]) to obtain the global existence, uniqueness and regularity of the solution to problem (1.1)-(1.3).

Lemma 2.1. Suppose that the initial data satisfy $\left(u_{0}, u_{1}\right)^{T} \in \mathcal{H}$. Then there exists a unique global mild solution $u$ to problem (1.1)-(1.3) such that

$$
\begin{gathered}
\left(u, u_{t}\right)^{T} \in C([0,+\infty) ; \mathcal{H}), \quad \partial_{\nu} u \in L_{l o c}^{2}(\Sigma), \quad u_{t} \in L_{l o c}^{2}(\Sigma), \quad \partial_{\nu} u+u+u_{t}=0 \text { on } \Gamma, \\
\mathcal{E}\left(u(t), u_{t}(t)\right)+\int_{0}^{t}\left\|u_{t}\right\|^{2} d \tau+\int_{0}^{t}\left\|u_{t}\right\|_{L^{2}(\Gamma)}^{2} d \tau=\mathcal{E}\left(u(0), u_{t}(0)\right),
\end{gathered}
$$

where $\Sigma=[0,+\infty) \times \Omega$. Furthermore, if $\left(u_{0}, u_{1}\right)^{T} \in \mathcal{D}$, then there exists a unique global classical solution $u$ to problem (1.1)-(1.3) such that

$$
\left(u, u_{t}\right)^{T} \in C([0,+\infty) ; \mathcal{D}) \cap C^{1}([0,+\infty) ; \mathcal{H}), \quad u_{t t} \in C\left([0,+\infty) ; L^{2}(\Omega)\right) .
$$


Proof. Notice that in the paper [7. (see also [17, 18]) the authors have used nonlinear semigroup theory to get the related result for a semilinear wave equation with critical exponent and nonlinear dissipative boundary conditions. Although in their setting, there is no interior damping term $u_{t}$ in equation (1.1), the proof is essentially the same. For the sake of the completeness and convenience of the reader, we give the sketch of the proof here. In what follows we adopt the notation used in [7.

First we introduce the Robin-Laplacian $\Delta_{R}: L^{2}(\Omega) \rightarrow L^{2}(\Omega)$. This is an unbounded operator with the domain

$$
D\left(\Delta_{R}\right)=\left\{u \in H^{2}(\Omega)\left|\partial_{\nu} u+u\right|_{\Gamma}=0\right\} .
$$

It is easy to see that this densely defined operator is injective and self-adjoint. Moreover, it can be extended to a continuous operator $\Delta_{R}: H^{1}(\Omega) \rightarrow H^{1}(\Omega)^{\prime}$ by

$$
\left(-\Delta_{R} u, v\right)=\int_{\Omega} \nabla u \cdot \nabla v d x+\int_{\Gamma} u v d S
$$

for all $v \in H^{1}(\Omega)$.

Next we introduce the Robin map $R: H^{s}(\Gamma) \rightarrow H^{s+(3 / 2)}(\Omega)$, which is defined as follows:

$$
R p=q \Leftrightarrow \Delta q=0 \text { in } \Omega, \partial_{\nu} q+q=p \text { on } \Gamma .
$$

As mentioned in [7, using the elliptic theory in [23], one can show that $R$ is continuous for $s \in \mathbb{R}$. The adjoint of the Robin map satisfies

$$
R^{*} \Delta_{R} v=-\left.v\right|_{\Gamma} \quad \text { for all } v \in H^{1}(\Omega) .
$$

Next we introduce a nonlinear operator $A$ with the domain $D(A)=\mathcal{D}$ by setting

$$
A\left(\begin{array}{c}
u \\
v
\end{array}\right)=\left(\begin{array}{c}
-v \\
-\Delta_{R}(u+R(\gamma v))+v
\end{array}\right)
$$

where $\gamma$ is the trace operator. Then it is easy to verify (see [7]) that for all $(u, v)^{T} \in \mathcal{D}$, $u+R(\gamma v) \in D\left(\Delta_{R}\right)$. In a similar manner as in [7, one can then check that $A$ is monotone on $\mathcal{H}$ and the range of $I+A$ is $\mathcal{H}$. Thus $A$ is a maximal monotone operator. Now we can rewrite the original problem as an abstract first-order evolution equation

$$
\frac{d}{d t}\left(\begin{array}{c}
u \\
u_{t}
\end{array}\right)+A\left(\begin{array}{c}
u \\
u_{t}
\end{array}\right)+\left(\begin{array}{c}
0 \\
f(x, u)
\end{array}\right)=0
$$

From the growth assumption $(\mathbf{F} 1)$ on $f(x, u)$ one can easily see that the operator $C\left(u, u_{t}\right)^{T}=(0, f(x, u))^{T}$ is locally Lipschitz continuous from $\mathcal{H}$ to $\mathcal{H}$. Hence, for $\left(u_{0}, u_{1}\right)^{T} \in \mathcal{H}$ there exists a unique local mild solution $\left(u, u_{t}\right)^{T} \in C\left(\left[0, t_{\text {max }}\right) ; \mathcal{H}\right)$, and for $\left(u_{0}, u_{1}\right)^{T} \in \mathcal{D}$ there exists a unique local classical solution $\left(u, u_{t}\right)^{T}$ on the interval $\left[0, t_{\max }\right.$ ) (refer to [37, Theorem 2.5.4 and Theorem 2.5.5). Moreover, if $t_{\max }<\infty$, we must have $\lim _{t \rightarrow t_{\max }}\left\|\left(u, u_{t}\right)^{T}\right\|_{\mathcal{H}}=+\infty$.

From the standard energy identity

$$
\mathcal{E}\left(u(t), u_{t}(t)\right)+\int_{0}^{t} \int_{\Gamma} u_{t}^{2} d S+\int_{0}^{t} \int_{\Omega} u_{t}^{2} d x=\mathcal{E}\left(u(0), u_{t}(0)\right),
$$


we know that the energy is nonincreasing. Following the similar steps in ([7, pp. 19121913) we can easily prove that there exists a constant $C_{1}$ depending on $\left\|\left(u(0), u_{t}(0)\right)^{T}\right\|_{\mathcal{H}}$ such that

$$
\mathcal{E}\left(u(0), u_{t}(0)\right) \leq C_{1}
$$

and also an estimate in the opposite direction

$$
\left\|\left(u(t), u_{t}(t)\right)^{T}\right\|_{\mathcal{H}} \leq C_{2}-C_{3},
$$

where $C_{2}$ is a positive constant depending on $\mathcal{E}\left(u(t), u_{t}(t)\right)$, and $C_{3}$ is a positive constant depending on $f$ and $\Omega$. Combining (2.9)-(2.11), we get

$$
\left\|\left(u(t), u_{t}(t)\right)^{T}\right\|_{\mathcal{H}} \leq C_{4}-C_{3},
$$

where $C_{4}$ is a positive constant depending only on $\left\|\left(u(0), u_{t}(0)\right)^{T}\right\|_{\mathcal{H}}$. This implies the following a priori estimate:

$$
\left\|\left(u, u_{t}\right)^{T}\right\|_{\mathcal{H}} \leq M \quad \text { for } t \in\left(0, t_{\max }\right),
$$

where $M$ depends only on the norm of initial data in $\mathcal{H}$, the measure of $\Omega$ and the nonlinear function $f$, and it does not depend on $t_{\max }$. Thus, this yields that $t_{\max }=+\infty$ (refer to [37, Theorem 2.5.5). Therefore, $\left(u, u_{t}\right)^{T} \in C([0,+\infty) ; \mathcal{H})$ and the uniform estimate

$$
\left\|\left(u, u_{t}\right)^{T}\right\|_{\mathcal{H}} \leq M \quad \text { for } t \geq 0
$$

holds. For $\left(u_{0}, u_{1}\right)^{T} \in \mathcal{D}$, since $\mathcal{H}$ is a Hilbert space, we deduce that the global mild solution is also a classical one (refer to [37, Theorem 2.5.4), i.e., $\left(u, u_{t}\right)^{T} \in C([0,+\infty) ; \mathcal{D}) \cap$ $C^{1}([0,+\infty) ; \mathcal{H})$.

The following lemma plays an important role in the subsequent proof.

Lemma 2.2. For initial data $\left(u_{0}, u_{1}\right)^{T} \in \mathcal{D}$, we have the following uniform estimate of the solution:

$$
\left\|\left(u(t), u_{t}(t)\right)^{T}\right\|_{\mathcal{D}} \leq C\left(\left\|\left(u_{0}, u_{1}\right)^{T}\right\|_{\mathcal{D}}\right) \quad \text { for } t \geq 0,
$$

where $C\left(\left\|\left(u_{0}, u_{1}\right)^{T}\right\|_{\mathcal{D}}\right)$ denotes a positive constant depending only on $\left\|\left(u_{0}, u_{1}\right)^{T}\right\|_{\mathcal{D}}$.

Proof. In the above lemma we have obtained the estimates

$$
\|u\|_{H^{1}(\Omega)} \leq C, \quad\left\|u_{t}\right\| \leq C \quad \forall t \geq 0,
$$

where $C$ is a positive constant depending on $\left\|\left(u_{0}, u_{1}\right)^{T}\right\|_{\mathcal{H}}$.

Let

$$
v=u_{t}
$$

then $\left(v, v_{t}\right)^{T} \in C([0,+\infty) ; \mathcal{H})$ and $v$ satisfies

$$
\left\{\begin{array}{l}
v_{t t}-\Delta v+v_{t}+f^{\prime}(x, u) v=0 \quad t>0, x \in \Omega \\
\partial_{\nu} v+v+v_{t}=0, \quad t>0, x \in \Gamma \\
\left.v\right|_{t=0}=u_{1},\left.\quad v_{t}\right|_{t=0}=\Delta u_{0}-u_{1}-f\left(x, u_{0}\right) .
\end{array}\right.
$$


Multiplying (2.16) by $v_{t}$ and $v$ respectively, integrating over $\Omega$ and using the boundary condition, we obtain

$$
\begin{aligned}
& \frac{d}{d t}\left(\frac{1}{2} \int_{\Omega} v_{t}^{2} d x+\frac{1}{2} \int_{\Omega}|\nabla v|^{2} d x+\frac{1}{2} \int_{\Gamma} v^{2} d S+\frac{1}{2} \int_{\Omega} f^{\prime}(x, u) v^{2} d x\right) \\
& +\int_{\Gamma} v_{t}^{2} d S+\int_{\Omega} v_{t}^{2} d x \\
= & \frac{1}{2} \int_{\Omega} f^{\prime \prime}(x, u) v^{3} d x
\end{aligned}
$$

and

$$
\begin{aligned}
& \frac{d}{d t}\left(\int_{\Omega} v v_{t} d x+\frac{1}{2} \int_{\Omega} v^{2} d x+\frac{1}{2} \int_{\Gamma} v^{2} d S\right)-\int_{\Omega} v_{t}^{2} d x+\int_{\Omega}|\nabla v|^{2} d x \\
& +\int_{\Gamma} v^{2} d S+\int_{\Omega} f^{\prime}(x, u) v^{2} d x=0 .
\end{aligned}
$$

Multiplying (2.18) by $\frac{1}{4}$, then summing with (2.17), we get

$$
\begin{aligned}
& \frac{d}{d t}\left(\frac{1}{2} \int_{\Omega} v_{t}^{2} d x+\frac{1}{2} \int_{\Omega}|\nabla v|^{2} d x+\frac{5}{8} \int_{\Gamma} v^{2} d S+\frac{1}{8} \int_{\Omega} v^{2} d x\right. \\
& \left.+\frac{1}{4} \int_{\Omega} v v_{t} d x+\frac{1}{2} \int_{\Omega} f^{\prime}(x, u) v^{2} d x\right) \\
& +\frac{3}{4} \int_{\Omega} v_{t}^{2} d x+\frac{1}{4} \int_{\Gamma} v^{2} d S+\int_{\Gamma} v_{t}^{2} d S+\frac{1}{4} \int_{\Omega}|\nabla v|^{2} d x+\frac{1}{4} \int_{\Omega} f^{\prime}(x, u) v^{2} d x \\
= & \frac{1}{2} \int_{\Omega} f^{\prime \prime}(x, u) v^{3} d x .
\end{aligned}
$$

By the assumption (F2), Hölder's inequality, the Sobolev imbedding theorem and (2.13), we have

$$
\left|\int_{\Omega} f^{\prime \prime}(x, u) v^{3} d x\right| \leq c \int_{\Omega}(1+|u|)\left|v^{3}\right| d x \leq C\left(1+\|u\|_{L^{6}}\right)\left\|v^{2}\right\|_{L^{3}}\|v\| \leq C\|v\|_{H^{1}}^{2}\|v\| .
$$

By the well-known Gagliardo-Nirenberg inequality (e.g., refer to [37]), we have

$$
\|v\|_{L^{3}} \leq C_{1}\|\nabla v\|^{\frac{1}{2}}\|v\|^{\frac{1}{2}}+C_{2}\|v\| .
$$

From (F2), (2.13), (2.21) and Young's inequality we can deduce that

$$
\left|\int_{\Omega} f^{\prime}(u) v^{2} d x\right| \leq C\left(\int_{\Omega} u^{2} v^{2} d x+\|v\|^{2}\right) \leq C\left(\|u\|_{L^{6}}^{2}\|v\|_{L^{3}}^{2}+C\right) \leq \varepsilon_{1}\|\nabla v\|^{2}+C,
$$

where $C$ is a positive constant depending only on $\left\|\left(u_{0}, u_{1}\right)\right\|_{\mathcal{H}}$ and $\varepsilon_{1}$. Let

$J(t):=\frac{1}{2} \int_{\Omega} v_{t}^{2} d x+\frac{1}{2} \int_{\Omega}|\nabla v|^{2} d x+\frac{5}{8} \int_{\Gamma} v^{2} d S+\frac{1}{8} \int_{\Omega} v^{2} d x+\frac{1}{4} \int_{\Omega} v v_{t} d x+\int_{\Omega} f^{\prime}(x, u) v^{2} d x$.

By the Cauchy-Schwarz inequality $\int_{\Omega} v v_{t} d x \leq \frac{1}{2}\|v\|^{2}+\frac{1}{2}\left\|v_{t}\right\|^{2}$, (2.20) and (2.22) after taking $\varepsilon_{1}$ small, it is easy to see that

$$
\frac{d}{d t} J(t)+\eta J(t) \leq C\|v\| J(t)+C\left(\left\|\left(u_{0}, u_{1}\right)^{T}\right\|_{\mathcal{H}}\right)
$$

where $\eta>0$ is a certain positive constant. 
Since $v=u_{t}$ satisfies

$$
\int_{0}^{+\infty}\|v\|^{2} d t<+\infty
$$

then we can see that

$$
\int_{\tau}^{t}\|v\| d s \leq\left(\int_{\tau}^{t}\|v\|^{2} d s\right)^{\frac{1}{2}}(t-\tau)^{\frac{1}{2}} \leq C(t-\tau)^{1 / 2}, \quad \forall t>\tau \geq 0 .
$$

Then it follows from a suitable version of the Gronwall inequality (see, for instance, Lemma 2.2 in 10 ) that

$$
J(t) \leq C\left(\left\|\left(u_{0}, u_{1}\right)^{T}\right\|_{\mathcal{D}}\right), \quad t \geq 0 .
$$

Thus, we can deduce from (2.25), (2.22) and the Cauchy-Schwarz inequality $\int_{\Omega} v v_{t} d x \leq$ $\frac{1}{2}\|v\|^{2}+\frac{1}{2}\left\|v_{t}\right\|^{2}$ that for $t \geq 0$,

$$
\left\|u_{t t}\right\|=\left\|v_{t}\right\| \leq C\left(\left\|\left(u_{0}, u_{1}\right)^{T}\right\|_{\mathcal{D}}\right), \quad\left\|u_{t}\right\|_{H^{1}}=\|v\|_{H^{1}} \leq C\left(\left\|\left(u_{0}, u_{1}\right)^{T}\right\|_{\mathcal{D}}\right) .
$$

For $u$, we reconsider (1.1), (1.2) as an elliptic boundary value problem:

$$
\left\{\begin{array}{l}
\Delta u=u_{t t}+u_{t}+f(x, u), \quad x \in \Omega \\
\partial_{\nu} u+u=-u_{t}, \quad x \in \Gamma .
\end{array}\right.
$$

By the regularity theory for elliptic problems we obtain

$$
\|u\|_{H^{2}(\Omega)} \leq C\left(\left\|u_{t t}\right\|+\left\|u_{t}\right\|+\|f(x, u)\|+\left\|u_{t}\right\|_{H^{\frac{1}{2}(\Gamma)}}\right) \leq C\left(\left\|\left(u_{0}, u_{1}\right)^{T}\right\|_{\mathcal{D}}\right) .
$$

Thus, we can conclude that

$$
\|u(t)\|_{H^{2}(\Omega)} \leq C, \quad\left\|u_{t}(t)\right\|_{H^{1}(\Omega)} \leq C, \quad\left\|u_{t t}(t)\right\| \leq C, \quad \text { for } t \geq 0,
$$

where $C$ is a constant that does not depend on $t$.

\section{Extended Simon-Lojasiewicz inequality. Let}

$$
E(u)=\frac{1}{2} \int_{\Omega}|\nabla u|^{2} d x .+\frac{1}{2} \int_{\Gamma} u^{2} d S+\int_{\Omega} F(x, u) d x .
$$

Lemma 3.1. Suppose that $\psi \in H^{2}(\Omega)$ is a strong solution to problem (1.6). Then $\psi$ is a critical point of the functional $E(u)$ in $H^{1}$. Conversely, if $\psi$ is a critical point of the functional $E(u)$ in $H^{1}$, then $\psi \in H^{2}(\Omega)$, and it is a strong solution to problem (1.6).

Proof. If $\psi \in H^{2}$ satisfies (1.6), then for any $v \in H^{1}(\Omega)$, it follows from (1.6) that

$$
\int_{\Omega}(-\triangle \psi+f(x, \psi)) v d x=0 .
$$

By integration by parts and the boundary condition in (1.6), we get

$$
\int_{\Omega}(\nabla \psi \cdot \nabla v+f(x, \psi) v) d x+\int_{\Gamma} \psi v d S=0,
$$

which, by a straightforward calculation, is just the following:

$$
\left.\frac{d E(\psi+\varepsilon v)}{d \varepsilon}\right|_{\varepsilon=0}=0 \text {. }
$$


Thus, $\psi$ is a critical point of $E(u)$. Conversely, if $\psi$ is a critical point of $E(u)$ in $H^{1}$, then (3.3) is satisfied. By the assumption $(\mathbf{F 1}), f(x, \psi) \in L^{2}(\Omega)$. Thus, by the regularity theory for the elliptic problem (1.6), $\psi \in H^{2}$ and it is a strong solution.

REMARK 3.2. By the bootstrap argument, $\psi$ is also a classical solution.

The following lemma claims that problem (1.6) admits at least a classical solution.

LEmma 3.3. The functional $E(u)$ has at least a minimizer $v \in H^{1}(\Omega)$ such that

$$
E(v)=\inf _{u \in H^{1}} E(u) .
$$

In other words, problem (1.6) admits at least a classical solution.

Proof. As proved in [7, assumption (F2) implies that there exist $\delta>0$ and $N=$ $N(\delta)>0$ such that

$$
F(x, s) \geq-\frac{\lambda-\delta}{2} s^{2} \quad \text { for }|s| \geq N .
$$

Let $M$ be a positive constant such that $f(x, z) / z+\lambda \geq 2 \delta$ for $|z| \geq M$ and certain $\delta>0$. Then we have

$$
\begin{aligned}
F(x, s)+\lambda \frac{s^{2}}{2} & =\int_{0}^{M}\left(\frac{f(x, z)}{z}+\lambda\right) z d z+\int_{M}^{s}\left(\frac{f(x, z)}{z}+\lambda\right) z d z \\
& \geq C+2 \delta\left(\frac{s^{2}}{2}-\frac{M^{2}}{2}\right) \geq \delta \frac{s^{2}}{2}
\end{aligned}
$$

for $s^{2} \geq \delta^{-1}\left(M^{2}-2 C\right)$. For negative $s$ one can repeat the same computation with $M$ replaced by $-M$. Now we have

$$
\int_{\Omega} F(x, u) d x=\int_{|u| \leq N} F(x, u) d x+\int_{|u|>N} F(x, u) d x \geq-\frac{\lambda-\delta}{2} \int_{\Omega} u^{2} d x+C(|\Omega|, f),
$$

where $C(|\Omega|, f)=|\Omega| \min _{|s| \leq N} F(x, s)$.

By the definition of $\lambda$ in (F2) we can deduce that

$E(u) \geq \frac{1}{2} \int_{\Omega}|\nabla u|^{2} d x+\frac{1}{2} \int_{\Gamma} u^{2} d S-\frac{\lambda-\delta}{2} \int_{\Omega} u^{2} d x+C(|\Omega|, f) \geq \frac{\delta}{2} \int_{\Omega} u^{2} d x+C(|\Omega|, f)$,

i.e., $E(u)$ is bounded from below. Therefore, there is a minimizing sequence $u_{n} \in H^{1}(\Omega)$ such that

$$
E\left(u_{n}\right) \rightarrow \inf _{u \in H^{1}} E(u) .
$$

Since $E$ can be written in the form

$$
E(u)=\frac{1}{2}\|u\|_{H^{1}}^{2}+\mathcal{F}(u)
$$

with

$$
\mathcal{F}(u)=\int_{\Omega} F(x, u) d x,
$$

it follows that $u_{n}$ is bounded in $H^{1}$. It turns out that there is a subsequence, still denoted by $u_{n}$, such that $u_{n}$ weakly converges to $v$ in $H^{1}$. Thus, $v \in H^{1}$. We infer from the Sobolev imbedding theorem $(n=3)$ that $u_{n}$ strongly converges to $v$ in $L^{4}$. It turns 
out from the assumption $(\mathbf{F} 1)$ that $\mathcal{F}\left(u_{n}\right) \rightarrow \mathcal{F}(v)$. Since $\|u\|_{H^{1}}^{2}$ is weakly lower semicontinuous, it follows from (3.9) that $E(v)=\inf _{u \in H^{1}} E(u)$. The proof is completed.

Now for $v \in C^{2}$, we consider the following linearized problem:

$$
\left\{\begin{array}{l}
L(v) h \equiv-\Delta h+f^{\prime}(x, v+\psi) h=0 \text { in } \Omega \\
\partial_{\nu} h+h=0 \text { on } \Gamma
\end{array}\right.
$$

where $\psi$ is a fixed critical point of $E(u)$ and we write

$$
u=v+\psi \text {. }
$$

The domain of $L(v)$ is

$$
\operatorname{Dom}(L(v))=\left\{h \in H^{2}\left|\partial_{\nu} h+h\right|_{\Gamma}=0\right\} .
$$

Lemma 3.4. $L(v)$ is a self-adjoint operator on $\operatorname{Dom}(L(v))$.

Proof. For any two functions $w_{1}, w_{2} \in \operatorname{Dom}(L(v))$, we have

$$
\begin{aligned}
& \left(L(v) w_{1}, w_{2}\right) \\
= & \int_{\Omega}\left(-\Delta w_{1}+f^{\prime}(v+\psi) w_{1}\right) w_{2} d x \\
= & \int_{\Omega}\left(\nabla w_{1} \cdot \nabla w_{2}+f^{\prime}(v+\psi) w_{1} w_{2}\right) d x+\int_{\Gamma} w_{1} w_{2} d S \\
= & \int_{\Omega}\left(-\Delta w_{2}+f^{\prime}(v+\psi) w_{2}\right) w_{1} d x \\
= & \left(w_{1}, L(v) w_{2}\right) .
\end{aligned}
$$

Thus we can easily see that $L(v)$ is a self-adjoint operator.

Associated with $L(v)$, we define the bilinear form $a\left(w_{1}, w_{2}\right)$ on $H^{1}(\Omega)$ as follows:

$$
a\left(w_{1}, w_{2}\right)=\int_{\Omega}\left(\nabla w_{1} \cdot \nabla w_{2}+f^{\prime}(v+\psi) w_{1} w_{2}\right) d x+\int_{\Gamma} w_{1} w_{2} d S .
$$

Then, the same as for the usual second-order elliptic operator, $L(v)+\lambda I$ with $\lambda>0$ being sufficiently large is invertible and its inverse is compact in $L^{2}$. It turns out from the Fredholm theorem that $\operatorname{Ker}(L(v))$ is finite-dimensional. It is well known that

$$
\operatorname{Ran}(L(v))=\left(\operatorname{Ker}\left(L^{*}(v)\right)\right)^{\perp} .
$$

Thus, we infer from Lemma 3.3 that

$$
\operatorname{Ran}(L(v))=(\operatorname{Ker}(L(v)))^{\perp}
$$

and

$$
\operatorname{Ran}(L(v)) \oplus \operatorname{Ker}(L(v))=L^{2}(\Omega) .
$$

Next we introduce two orthogonal projections $\Pi_{K}$ and $\Pi_{R}$ in $L^{2}(\Omega)$; namely, $\Pi_{K}$ is the projection onto the kernel of $L(0)$ while $\Pi_{R}$ is the projection onto the range of $L(0)$. Then we have the following result. 
Lemma 3.5. For

$$
L(0) w=f_{R}
$$

with $f_{R} \in \Pi_{R} L^{2}(\Omega)$, there exists a unique solution $w_{R} \in H^{2}(\Omega) \cap \Pi_{R} L^{2}(\Omega)$ and the following estimate holds:

$$
\left\|w_{R}\right\|_{H^{2}(\Omega)} \leq C\left\|f_{R}\right\| .
$$

Proof. By the Fredholm alternative theory and the regularity theorem for the elliptic operator, we have a function $w \in \operatorname{Dom}(L(0)) \cap H^{2}$ such that $L(0) w=f_{R}$. Moreover $w$ is unique if we require $w \in(\operatorname{Ker} L(0))^{\perp}$, and (3.19) follows from the elliptic regularity theory.

Let $\mathcal{L}(v): \operatorname{Dom}(L(v)) \rightarrow L^{2}(\Omega)$ be defined as follows:

$$
\mathcal{L}(v) w=\Pi_{K} w+L(v) w
$$

where, as indicated previously, $\Pi_{K}$ is the project operator from $L^{2}$ onto $\operatorname{Ker}(L(0))$. Then it follows from Lemma 3.3 and Lemma 3.4 that $\mathcal{L}(0)$ is one-to-one and onto. Moreover, its inverse $\mathcal{L}^{-1}(0)$ is a linear bounded operator from $L^{2}(\Omega)$ to $H^{2}(\Omega)$.

Lemma 3.6. There exists a small positive constant $\beta<1$ depending on $\psi$ such that if $v \in H^{2}(\Omega),\|v\|_{H^{1}} \leq \beta$, and $g \in L^{2}(\Omega)$, then the problem

$$
\left\{\begin{array}{l}
\mathcal{L}(v) w=g \text { in } \Omega \\
\partial_{\nu} w+w=0 \text { on } \Gamma
\end{array}\right.
$$

has a unique solution $w \in H^{2}(\Omega)$. Moreover, the following estimate holds:

$$
\|w\|_{H^{2}} \leq C\|g\| .
$$

Proof. By Lemma 3.4, $\mathcal{L}(0)$ is invertible, and its inverse $\mathcal{L}^{-1}(0)$ is a linear bounded operator from $L^{2}(\Omega)$ into $H^{2}(\Omega)$. To see the solvability of (3.21), we rewrite (3.21) in the form:

$$
\left(\mathcal{L}^{-1}(0)(\mathcal{L}(v)-\mathcal{L}(0))+I\right) w=\mathcal{L}^{-1}(0) g .
$$

By the definition, $(\mathcal{L}(v)-\mathcal{L}(0)) w=\left(f^{\prime}(x, v+\psi)-f^{\prime}(x, \psi)\right) w$. Since $n=3$, we infer from the Sobolev imbedding theorem and (F1) that for $\|v\|_{H^{1}} \leq \beta<1$,

$$
\begin{aligned}
\left\|\left(f^{\prime}(x, v+\psi)-f^{\prime}(x, \psi)\right) w\right\| & =\left\|f^{\prime \prime}(x, \psi+\zeta v) v w\right\| \\
& \leq\left(C_{1}\|v\|+C_{2}\|v\|_{L^{4}}^{2}\right)\|w\|_{L^{\infty}} \\
& \leq C\|v\|_{H^{1}(\Omega)}\|w\|_{H^{2}(\Omega)},
\end{aligned}
$$

where $\zeta \in(0,1)$.

Therefore, it follows that when $\beta$ is sufficiently small, $\mathcal{L}^{-1}(0)(\mathcal{L}(v)-\mathcal{L}(0))$ is a contraction from $H^{2}(\Omega)$ into $H^{2}(\Omega)$ :

$$
\left\|\mathcal{L}^{-1}(0)(\mathcal{L}(v)-\mathcal{L}(0))\right\|_{L\left(H^{2}, H^{2}\right)} \leq \frac{1}{2} .
$$

By the contraction mapping theorem, (3.21) is uniquely solvable, which implies that when $\|v\|_{H^{1}} \leq \beta<1, \mathcal{L}(v)$ is invertible, and (3.22) holds. Thus, the lemma is proved. 
We now prove the following generalized Simon-Lojasiewicz inequality, which extends the original one by Simon [31] for the second-order nonlinear parabolic equation, and also the one by Jendoubi [16 for the semilinear wave equation with Dirichlet boundary condition. In 34, we have used a similar type of inequality to prove the convergence to equilibrium for the Cahn-Hilliard equation with dynamic boundary conditions.

Lemma 3.7. Let $\psi$ be a critical point of $E(u)$. Then there exist constants $\theta \in\left(0, \frac{1}{2}\right)$ and $\beta_{0}>0$ depending on $\psi$ such that for $\forall u \in H^{2}(\Omega),\|u-\psi\|_{H^{2}}<\beta_{0} \leq \beta$, we have

$$
\|-\Delta u+f(x, u)\|+\left\|\partial_{\nu} u+u\right\|_{L^{2}(\Gamma)} \geq|E(u)-E(\psi)|^{1-\theta} .
$$

Proof. As before, we denote $u=v+\psi$ and let

$$
M(v)=-\Delta(v+\psi)+f(x, v+\psi) .
$$

Then $M(v)$ maps $v \in H^{2}(\Omega)$ into $L^{2}(\Omega)$.

Let $\mathcal{N}$ be a nonlinear operator from $H^{2}(\Omega)$ into $L^{2}(\Omega)$ defined as follows:

$$
\mathcal{N}(v)=\Pi_{K} v+M(v) .
$$

Hence, $\mathcal{N}(v)$ is holomorphic and its Fréchet derivative is $\mathcal{L}(v)$ :

$$
D \mathcal{N}(v) h=\mathcal{L}(v) h .
$$

Since $\mathcal{L}(v)$ is invertible for $\|v\|_{H^{1}} \leq \beta$, by the abstract implicit function theorem (see, e.g., Nirenberg [25]), there exist neighborhoods of the origin $W_{1}(0) \subset H^{2}(\Omega)$ and $W_{2}(0) \subset$ $L^{2}(\Omega)$, and a holomorphic inverse projection $\Psi$ of $\mathcal{N}$ such that $\Psi: W_{2}(0) \rightarrow W_{1}(0)$ is a one-to-one and onto mapping. Moreover,

$$
\begin{array}{ll}
\mathcal{N}(\Psi(g))=g & \forall g \in W_{2}(0), \\
\Psi(\mathcal{N}(v))=v & \forall v \in W_{1}(0),
\end{array}
$$

and

$$
\begin{aligned}
& \left\|\Psi\left(g_{1}\right)-\Psi\left(g_{2}\right)\right\|_{H^{2}} \leq C\left\|g_{1}-g_{2}\right\| \quad \forall g_{1}, g_{2} \in W_{2}(0), \\
& \left\|\mathcal{N}\left(v_{1}\right)-\mathcal{N}\left(v_{2}\right)\right\| \leq C\left\|v_{1}-v_{2}\right\|_{H^{2}} \quad \forall v_{1}, v_{2} \in W_{1}(0) .
\end{aligned}
$$

It follows from $\mathcal{N}[\Psi(g)]=g$ that $D \mathcal{N} \cdot D \Psi=I$. Therefore, $D \Psi(g)=\mathcal{L}^{-1}(\Psi(g))$. We infer from Lemma 3.5 that

$$
\|D \Psi\|_{L\left(L^{2}, H^{2}\right)} \leq C .
$$

Let $\phi_{1}, \ldots, \phi_{m}$ be the orthogonal unit vectors spanning $\operatorname{Ker} L(0)$. Since $\Psi$ is holomorphic, it turns out that

$$
\Gamma(\xi):=E\left(\Psi\left(\sum_{i=1}^{m} \xi_{i} \phi_{i}\right)+\psi\right)
$$

is analytic for small $|\xi|$ such that $\Pi_{K} v=\sum_{i=1}^{m} \xi_{i} \phi_{i} \in W_{2}(0)$.

In what follows we will show that for any $v$ satisfying $\Pi_{K} v=\sum_{i=1}^{m} \xi_{i} \phi_{i} \in W_{2}(0)$, we have

$$
|\nabla \Gamma(\xi)| \leq C\left(\|M(v)\|+\left\|\partial_{\nu} v+v\right\|_{L^{2}(\Gamma)}\right) .
$$


Indeed, a straightforward calculation, using (3.35) and integration by parts, yields

$$
\begin{aligned}
\frac{\partial \Gamma(\xi)}{\partial \xi_{i}}= & \int_{\Omega} M\left(\Psi\left(\Pi_{K} v\right)\right) D \Psi\left(\Pi_{K} v\right) \phi_{i} d x \\
& +\int_{\Gamma}\left(\partial_{\nu} \Psi\left(\Pi_{K} v\right)+\Psi\left(\Pi_{K} v\right)\right) D \Psi\left(\Pi_{K} v\right) \phi_{i} d S \\
:= & I_{1}+I_{2} .
\end{aligned}
$$

For the first part on the right-hand side of (3.37), we have

$$
\begin{aligned}
\left|I_{1}\right| & \leq\left\|M\left(\Psi\left(\Pi_{K} v\right)\right)\right\| \cdot\left\|D \Psi\left(\Pi_{K} v\right) \phi_{i}\right\| \\
& \leq C\left\|M\left(\Psi\left(\Pi_{K} v\right)\right)\right\| \\
& \leq C\left(\left\|M\left(\Psi\left(\Pi_{K} v\right)\right)-M(v)\right\|+\|M(v)\|\right) .
\end{aligned}
$$

Recalling the definition of $\Psi$ and noticing that $v=\Psi\left(\Pi_{K} v+M(v)\right)$, and $L(v)$ is a continuous operator from $H^{2}(\Omega)$ to $L^{2}(\Omega)$, we have

$$
\begin{aligned}
& \left\|M\left(\Psi\left(\Pi_{K} v\right)\right)-M(v)\right\| \\
\leq & \left\|\int_{0}^{1} L\left(t v+(1-t) \Psi\left(\Pi_{K} v\right)\right)\left(\Psi\left(\Pi_{K} v+M(v)\right)-\Psi\left(\Pi_{K} v\right)\right) d t\right\| \\
\leq & C\left\|\Psi\left(\Pi_{K} v+M(v)\right)-\Psi\left(\Pi_{K} v\right)\right\|_{H^{2}} \\
= & C\left\|\int_{0}^{1} D \Psi\left(\Pi_{K} v+t M(v)\right) M(v) d t\right\|_{H^{2}} .
\end{aligned}
$$

From Lemma 3.5, and $\|D \Psi\|_{L\left(L^{2}, H^{2}\right)} \leq C$ we infer that

$$
\left\|M\left(\Psi\left(\Pi_{K} v\right)\right)-M(v)\right\| \leq C\|M(v)\| .
$$

The combination of (3.38) with (3.40) yields

$$
\left|I_{1}\right| \leq C\|M(v)\|
$$

and

$$
\left\|\Psi\left(\Pi_{K} v\right)-v\right\|_{H^{2}(\Omega)}=\left\|\Psi\left(\Pi_{K} v\right)-\Psi\left(\Pi_{K} v+M(v)\right)\right\|_{H^{2}(\Omega)} \leq C\|M(v)\| .
$$

Next for $I_{2}$, using the trace theorem and applying (3.34), (3.42), we get

$$
\begin{aligned}
\left|I_{2}\right| & \leq\left\|\partial_{\nu} \Psi\left(\Pi_{K} v\right)+\Psi\left(\Pi_{K} v\right)\right\|_{L^{2}(\Gamma)}\left\|D \Psi\left(\Pi_{K} v\right) \phi_{i}\right\|_{L^{2}(\Gamma)} \\
& \leq C\left(\left\|\partial_{\nu}\left(\Psi\left(\Pi_{K} v\right)-v\right)+\left(\Psi\left(\Pi_{K} v\right)-v\right)\right\|_{L^{2}(\Gamma)}+\left\|\partial_{\nu} v+v\right\|_{L^{2}(\Gamma)}\right) \\
& \leq C\left(\left\|\partial_{\nu}\left(\Psi\left(\Pi_{K} v\right)-v\right)\right\|_{H^{\frac{1}{2}(\Gamma)}}+\left\|\Psi\left(\Pi_{K} v\right)-v\right\|_{H^{\frac{1}{2}(\Gamma)}}+\left\|\partial_{\nu} v+v\right\|_{L^{2}(\Gamma)}\right) \\
& \leq C\left(\left\|\Psi\left(\Pi_{K} v\right)-v\right\|_{H^{2}(\Omega)}+\left\|\partial_{\nu} v+v\right\|_{L^{2}(\Gamma)}\right) \\
& \leq C\left(\|M(v)\|+\left\|\partial_{\nu} v+v\right\|_{L^{2}(\Gamma)}\right) .
\end{aligned}
$$

The combination of (3.43) with (3.38) yields that for $1 \leq i \leq m$,

$$
\left|\frac{\partial \Gamma(\xi)}{\partial \xi_{i}}\right| \leq C\left(\|M(v)\|+\left\|\partial_{\nu} v+v\right\|_{L^{2}(\Gamma)}\right) .
$$

It turns out that

$$
|\nabla \Gamma(\xi)|=\left(\sum_{i=1}^{m}\left|\frac{\partial \Gamma(\xi)}{\partial \xi_{i}}\right|^{2}\right)^{\frac{1}{2}} \leq C\left(\|M(v)\|+\left\|\partial_{\nu} v+v\right\|_{L^{2}(\Gamma)}\right) .
$$


We now proceed to estimate $\left|E\left(\Psi\left(\Pi_{K} v\right)+\psi\right)-E(v+\psi)\right|$. By the Newton-Leibniz formula, we have

$$
\begin{aligned}
& \left|E\left(\Psi\left(\Pi_{K} v\right)+\psi\right)-E(v+\psi)\right| \\
\leq & \left|\int_{0}^{1} \int_{\Omega} M\left(v+t\left(\Psi\left(\Pi_{K} v\right)-v\right)\right)\left(\Psi\left(\Pi_{K} v\right)-v\right) d x d t\right| \\
& +\left|\int_{0}^{1} \int_{\Gamma}\left[\partial_{\nu}\left(v+t\left(\Psi\left(\Pi_{K} v\right)-v\right)\right)+\left(v+t\left(\Psi\left(\Pi_{K} v\right)-v\right)\right)\right]\left(\Psi\left(\Pi_{K} v\right)-v\right) d S d t\right| \\
:= & I_{3}+I_{4} .
\end{aligned}
$$

Using $v=\Psi\left(M(v)+\Pi_{K} v\right)$ and referring to (3.40), for $I_{3}$ we have

$$
\begin{aligned}
I_{3} & \leq \max _{0 \leq t \leq 1}\left\|M\left(v+t\left(\Psi\left(\Pi_{K} v\right)-v\right)\right)\right\| \cdot\left\|\Psi\left(\Pi_{K} v\right)-v\right\| \\
& \leq C\|M(v)\|\left(\max _{0 \leq t \leq 1}\left\|M\left(v+t\left(\Psi\left(\Pi_{K} v\right)-v\right)\right)-M(v)\right\|+\|M(v)\|\right) \\
& \leq C\|M(v)\|\left(C \max _{0 \leq t \leq 1}\left\|v+t\left(\Psi\left(\Pi_{K} v\right)-v\right)-v\right\|_{H^{2}}+\|M(v)\|\right) \\
& \leq C\|M(v)\|^{2} .
\end{aligned}
$$

By the Hölder inequality and the trace theorem, we have the following estimate for $I_{4}$ :

$$
\begin{aligned}
I_{4} \leq & \max _{0 \leq t \leq 1}\left(\left\|\partial_{\nu} v+v\right\|_{L^{2}(\Gamma)}+t\left\|\partial_{\nu}\left(\Psi\left(\Pi_{K} v\right)-v\right)+\left(\Psi\left(\Pi_{K} v\right)-v\right)\right\|_{L^{2}(\Gamma)}\right) \\
& \times\left\|\Psi\left(\Pi_{K} v\right)-v\right\|_{L^{2}(\Gamma)} \\
\leq & C\left(\left\|\partial_{\nu} v+v\right\|_{L^{2}(\Gamma)}+\left\|\Psi\left(\Pi_{K} v\right)-v\right\|_{H^{2}}\right)\left\|\Psi\left(\Pi_{K} v\right)-v\right\|_{H^{2}}
\end{aligned}
$$

The combination of (3.48) with (3.42) yields

$$
I_{4} \leq C\left(\left\|\partial_{\nu} v+v\right\|_{L^{2}(\Gamma)}+\|M(v)\|\right)\|M(v)\| .
$$

Finally, from (3.46)-(3.49) we deduce the following estimate on $\mid E\left(\Psi\left(\Pi_{K} v\right)+\psi\right)-$ $E(v+\psi) \mid:$

$$
\begin{aligned}
& \left|E\left(\Psi\left(\Pi_{K} v\right)+\psi\right)-E(v+\psi)\right| \\
\leq & C\left(\left\|\partial_{\nu} v+v\right\|_{L^{2}(\Gamma)}+\|M(v)\|\right)\|M(v)\| \\
\leq & C\left(\left\|\partial_{\nu} v+v\right\|_{L^{2}(\Gamma)}+\|M(v)\|\right)^{2} .
\end{aligned}
$$

Since $\Gamma(\xi)=E\left(\Psi\left(\Pi_{K} v\right)+\psi\right): \mathbb{R}^{m} \rightarrow \mathbb{R}$ is real analytic for small $|\xi|$ and $\nabla \Gamma(0)=0$, we have the following Lojasiewicz inequality on analytic functions defined on $\mathbb{R}^{m}$ (see [20]-22]): for $|\xi| \leq \beta_{1} \leq \beta$,

$$
|\nabla \Gamma(\xi)| \geq|\Gamma(\xi)-\Gamma(0)|^{1-\theta^{\prime}}
$$


where $\theta^{\prime} \in\left(0, \frac{1}{2}\right)$. Thus from (3.45), (3.51) and (3.50) we infer that

$$
\begin{aligned}
& C\left(\|M(v)\|+\left\|\partial_{\nu} v+v\right\|_{L^{2}(\Gamma)}\right) \\
\geq & |\nabla \Gamma(\xi)| \geq|\Gamma(\xi)-\Gamma(0)|^{1-\theta^{\prime}} \\
= & |\Gamma(\xi)-E(v+\psi)+E(v+\psi)-\Gamma(0)|^{1-\theta^{\prime}} \\
\geq & \frac{1}{2}|E(v+\psi)-\Gamma(0)|^{1-\theta^{\prime}}-C_{1}|\Gamma(\xi)-E(v+\psi)|^{1-\theta^{\prime}} \\
\geq & \frac{1}{2}|E(v+\psi)-\Gamma(0)|^{1-\theta^{\prime}}-C_{1}\left(\left\|\partial_{\nu} v+v\right\|_{L^{2}(\Gamma)}+\|M(v)\|\right)^{2\left(1-\theta^{\prime}\right)} .
\end{aligned}
$$

Hence, we see

$$
\begin{aligned}
& |E(v+\psi)-\Gamma(0)|^{1-\theta^{\prime}} \\
\leq \quad & \left(\left\|\partial_{\nu} v+v\right\|_{L^{2}(\Gamma)}+\|M(v)\|\right) \\
& \times\left(2 C+2 C_{1}\left(\left\|\partial_{\nu} v+v\right\|_{L^{2}(\Gamma)}+\|M(v)\|\right)^{2\left(1-\theta^{\prime}\right)-1}\right) .
\end{aligned}
$$

Since $0<\theta^{\prime}<\frac{1}{2}, 2\left(1-\theta^{\prime}\right)-1>0$, we can choose smaller $\beta_{2}<\beta_{1}$ such that when $\|v\|_{H^{2}(\Omega)} \leq \beta_{2}$,

$$
\left(\left\|\partial_{\nu} v+v\right\|_{L^{2}(\Gamma)}+\|M(v)\|\right)^{2\left(1-\theta^{\prime}\right)-1} \leq 1 .
$$

Then it follows from (3.53) that

$$
|E(v+\psi)-\Gamma(0)|^{1-\theta^{\prime}} \leq C\left(\left\|\partial_{\nu} v+v\right\|_{L^{2}(\Gamma)}+\|M(v)\|\right) .
$$

Next we choose $\varepsilon, 0<\varepsilon<\theta^{\prime}$ and $\beta_{0}<\beta_{2}$ such that for $\|v\|_{H^{2}}<\beta_{0}$,

$$
\frac{1}{C}|E(v+\psi)-\Gamma(0)|^{-\varepsilon} \geq 1 \text {. }
$$

Setting $\theta=\theta^{\prime}-\varepsilon \in\left(0, \frac{1}{2}\right)$, we finally have for $\|v\|_{H^{2}}<\beta_{0}$,

$$
\left\|\partial_{\nu} v+v\right\|_{L^{2}(\Gamma)}+\|M(v)\| \geq|E(v+\psi)-\Gamma(0)|^{1-\theta}=|E(u)-E(\psi)|^{1-\theta} .
$$

which is exactly (3.26). Thus, the lemma is proved.

In order to prove our Theorem 1.1, we need the following modification of the previous lemma in which we only need smallness of $\|u-\psi\|$ in $H^{1}$ norm, which is crucial for hyperbolic equations.

Lemma 3.8. Let $\psi$ be a critical point of $E(u)$. Then there exist constants $\widetilde{\theta} \in\left(0, \frac{1}{2}\right)$ and $\widetilde{\beta}_{0}>0$ depending on $\psi$ such that for $\forall u \in H^{2}(\Omega)$, we have

$$
\|-\Delta u+f(x, u)\|+\left\|\partial_{\nu} u+u\right\|_{H^{\frac{1}{2}(\Gamma)}} \geq|E(u)-E(\psi)|^{1-\theta}
$$

provided $\|u-\psi\|_{H^{1}(\Omega)}<\widetilde{\beta_{0}}$.

Proof. We consider the following two cases:

(i) If $\|u-\psi\|_{H^{2}(\Omega)}<\beta_{0}$, where $\beta_{0}$ is the constant appearing in Lemma 3.6, then we have

$$
\|-\Delta u+f(x, u)\|+\left\|\partial_{\nu} u+u\right\|_{L^{2}(\Gamma)} \geq|E(u)-E(\psi)|^{1-\theta} .
$$

Since $\|w\|_{H^{\frac{1}{2}(\Gamma)}} \geq\|w\|_{L^{2}(\Gamma)}, \forall w \in H^{1}(\Omega)$, it is easy to see that

$$
\|-\Delta u+f(x, u)\|+\left\|\partial_{\nu} u+u\right\|_{H^{\frac{1}{2}(\Gamma)}} \geq|E(u)-E(\psi)|^{1-\theta} .
$$


(ii) If $\|u-\psi\|_{H^{2}(\Omega)} \geq \beta_{0}$, then for $v=u-\psi$, from the regularity theory for elliptic problems we get

$$
\|v\|_{H^{2}} \leq C\left(\|\Delta v\|+\left\|\partial_{\nu} v+v\right\|_{H^{\frac{1}{2}(\Gamma)}}\right),
$$

where $C$ is a constant independent of $v$.

By $H^{1}(\Omega) \hookrightarrow L^{6}(\Omega)(n=3)$, there exists $\widetilde{\beta_{0}}>0$ depending on $\psi$ such that $\forall u$, $\|u-\psi\|_{H^{1}(\Omega)}<\widetilde{\beta}_{0}$,

and

$$
\|f(x, u)-f(x, \psi)\|<\frac{\beta_{0}}{2 C}
$$

$$
|E(u)-E(\psi)|^{1-\theta}<\frac{\beta_{0}}{2 C}
$$

Noticing that $\psi$ satisfies (1.6), we deduce from (3.61), (3.62) and (3.63) that

$$
\begin{aligned}
& \|-\Delta u+f(x, u)\|+\left\|\partial_{\nu} u+u\right\|_{H^{\frac{1}{2}}(\Gamma)} \\
= & \|-\Delta v+f(x, u)-f(x, \psi)\|+\left\|\partial_{\nu} v+v\right\|_{H^{\frac{1}{2}}(\Gamma)} \\
\geq & \left(\|\Delta v\|+\left\|\partial_{\nu} v+v\right\|_{H^{\frac{1}{2}}(\Gamma)}\right)-\|f(x, u)-f(x, \psi)\| \\
\geq & \frac{1}{C}\|v\|_{H^{2}}-\|f(x, u)-f(x, \psi)\| \\
> & \frac{\beta_{0}}{2 C}>|E(u)-E(\psi)|^{1-\theta} .
\end{aligned}
$$

Thus the lemma is proved.

4. Proof of Theorem 1.1. The proof consists of several steps.

Step 1. The $\omega$-limit set of $\left(u_{0}, u_{1}\right)^{T} \in \mathcal{H}$ is defined as follows:

$$
\begin{aligned}
\omega\left(u_{0}, u_{1}\right)=\left\{(\psi(x), \phi(x))^{T} \in \mathcal{H} \mid\right. \\
\left.\quad \exists t_{n} \rightarrow+\infty \text { such that }\left(u\left(x, t_{n}\right), u_{t}\left(x, t_{n}\right)\right)^{T} \rightarrow(\psi(x), \phi(x))^{T} \text { in } \mathcal{H}\right\} .
\end{aligned}
$$

Then we have

Lemma 4.1. For any $\left(u_{0}, u_{1}\right)^{T} \in \mathcal{D}$, the $\omega$-limit set of $\left(u_{0}, u_{1}\right)^{T}$ is a nonempty compact connected subset in $\mathcal{H}$. Furthermore,

(i) It is invariant under the nonlinear semigroup $S(t)$ defined by the solution $(u(x, t)$, $\left.u_{t}(x, t)\right)^{T}$, i.e., $S(t) \omega\left(u_{0}, u_{1}\right)=\omega\left(u_{0}, u_{1}\right)$ for all $t \geq 0$.

(ii) $E(u)$ is constant on $\omega\left(u_{0}, u_{1}\right)$. Moreover, $\omega\left(u_{0}, u_{1}\right)$ consists of equilibria.

Proof. For the proof one can refer to Theorem 5.2 in 7 .

Thus it follows that every element in $\omega\left(u_{0}, u_{1}\right)$ has the form $(\psi(x), 0)^{T}$, where $\psi(x)$ is a solution to problem (1.6), and

$$
\left\|u_{t}\right\| \rightarrow 0, \quad \text { as } t \rightarrow+\infty .
$$

Step 2. After the previous preparations, we now proceed to finish the proof of Theorem 1.1, following a simple argument introduced in 15] in which the key observation is that after a certain time $t_{0}$, the solution $u$ will fall into the small neighborhood of $\psi$ and stay there forever. 
As mentioned in the Introduction section, for the semilinear wave equation, there is a term $u_{t t}$ in the equation (1.1), being different from the parabolic equation. It turns out that we cannot directly apply the extended Simon-Lojasiewicz inequality derived in the previous section. Instead, we have to introduce an auxiliary function. Now let $\varepsilon$ be a small positive real number, and define

$$
\begin{aligned}
H(t)= & \frac{1}{2} \int_{\Omega} u_{t}^{2} d x+E(u)+\varepsilon \int_{\Omega}[-\Delta u+f(x, u)] u_{t} d x+\varepsilon \int_{\Omega}\left|\nabla u_{t}\right|^{2} d x \\
& +\varepsilon \int_{\Omega}|-\Delta u+f(x, u)|^{2} d x+\varepsilon \int_{\Omega} f^{\prime}(x, u)\left|u_{t}\right|^{2} d x+\frac{3}{2} \varepsilon\left\|u_{t}\right\|_{L^{2}(\Gamma)}^{2}
\end{aligned}
$$

Then $H(t)$ is well defined for all $t \geq 0$ as a sequence of Lemma 2.2 and $H(t)$ is bounded from below. We have for $t \geq 0$,

$$
\begin{aligned}
\frac{d H}{d t}= & -\left\|u_{t}\right\|^{2}-\left\|u_{t}\right\|_{L^{2}(\Gamma)}^{2}+\varepsilon \int_{\Omega}[-\Delta u+f(x, u)]_{t} u_{t} d x+\varepsilon \int_{\Omega}[-\Delta u+f(x, u)] u_{t t} d x \\
& +2 \varepsilon \int_{\Omega} \nabla u_{t} \cdot \nabla u_{t t} d x+2 \varepsilon \int_{\Omega}[\Delta u-f(x, u)]_{t}[\Delta u-f(x, u)] d x \\
& +\varepsilon \int_{\Omega} f^{\prime \prime}(x, u)\left|u_{t}\right|^{2} u_{t} d x+2 \varepsilon \int_{\Omega} f^{\prime}(x, u) u_{t} u_{t t} d x+3 \varepsilon \int_{\Gamma} u_{t} u_{t t} d S \\
= & -\left\|u_{t}\right\|^{2}-\left\|u_{t}\right\|_{L^{2}(\Gamma)}^{2}-\varepsilon\left\|\nabla u_{t}\right\|^{2}-\varepsilon \int_{\Omega} f^{\prime}(x, u) u_{t}^{2} d x-\varepsilon\|-\Delta u+f(x, u)\|^{2} \\
& +\varepsilon \int_{\Omega}[\Delta u-f(x, u)] u_{t} d x-2 \varepsilon\left\|u_{t t}\right\|_{L^{2}(\Gamma)}+\varepsilon \int_{\Omega} f^{\prime \prime}(x, u)\left|u_{t}\right|^{2} u_{t} d x \\
& -\varepsilon\left\|u_{t}\right\|_{L^{2}(\Gamma)}^{2} .
\end{aligned}
$$

Using the Cauchy-Schwarz inequality for the term $\int_{\Omega}[\Delta u-f(x, u)] u_{t} d x$ we find that

$$
\begin{aligned}
\frac{d H}{d t} \leq & \int_{\Omega}\left[-\frac{1}{2}-\varepsilon f^{\prime}(x, u)+\varepsilon f^{\prime \prime}(x, u) u_{t}\right]\left|u_{t}\right|^{2} d x-\frac{\varepsilon}{2}\|-\Delta u+f(x, u)\|^{2} \\
& -(1+\varepsilon)\left\|u_{t}\right\|_{L^{2}(\Gamma)}^{2}-\varepsilon\left\|\nabla u_{t}\right\|^{2} .
\end{aligned}
$$

We note that $u \in H^{2}(\Omega) \hookrightarrow L^{\infty}(\Omega)(n=3)$ and then by Lemma $2.2, f^{\prime}(x, u)$ and $f^{\prime \prime}(x, u)$ remain bounded. By the Gagliardo-Nirenberg inequality

$$
\left\|u_{t}\right\|_{L^{3}}^{3} \leq C_{1}\left\|\nabla u_{t}\right\|^{\frac{3}{2}}\left\|u_{t}\right\|^{\frac{3}{2}}+C_{2}\left\|u_{t}\right\|^{3} .
$$

Then we have

$$
\int_{\Omega} f^{\prime \prime}(x, u) u_{t}^{3} d x \leq C_{0}\left\|u_{t}\right\|_{L^{3}}^{3} \leq C_{0}\left(\varepsilon_{1}\left\|\nabla u_{t}\right\|^{2}+C\left(\varepsilon_{1}\right)\left\|u_{t}\right\|^{6}+C_{2}\left\|u_{t}\right\|^{3}\right) .
$$

Taking $\varepsilon_{1}$ small such that $C_{0} \varepsilon_{1}<\frac{1}{2}$, and taking $\varepsilon$ small such that $\left|\varepsilon f^{\prime}(x, u)\right|<\frac{1}{4}$, we can conclude from (4.1) and also the trace theorem that there exists $C_{3}>0, \widetilde{C}_{3}>0$ and 
$T_{1}>0$ such that for all $t \geq T_{1}>0$,

$$
\begin{aligned}
& \frac{d}{d t} H(t) \\
\leq & -C_{3}\left(\left\|u_{t}\right\|^{2}+\|-\Delta u+f(x, u)\|^{2}+\left\|\nabla u_{t}\right\|^{2}+\left\|u_{t}\right\|_{L^{2}(\Gamma)}^{2}\right) \\
\leq & -\frac{C_{3}}{4}\left(\left\|u_{t}\right\|+\|-\Delta u+f(x, u)\|+\left\|\nabla u_{t}\right\|+\left\|u_{t}\right\|_{L^{2}(\Gamma)}\right)^{2} \\
\leq & -\widetilde{C}_{3}\left(\left\|u_{t}\right\|+\|-\Delta u+f(x, u)\|+\left\|\nabla u_{t}\right\|+\left\|u_{t}\right\|_{L^{2}(\Gamma)}+\left\|u_{t}\right\|_{H^{\frac{1}{2}}(\Gamma)}\right)^{2},
\end{aligned}
$$

i.e., $H(t)$ is decreasing on $\left[T_{1},+\infty\right)$. It follows that as time goes to $+\infty, H(t)$ has a finite limit. Since $(\psi(x), 0)^{T} \in \omega\left(u_{0}, u_{1}\right)$, there is a sequence $t_{n}, t_{n} \rightarrow+\infty$ such that

$$
u\left(x, t_{n}\right) \rightarrow \psi(x)
$$

in $H^{1}(\Omega)$. It turns out that

$$
E\left(t_{n}\right) \rightarrow E(\psi) .
$$

It easily follows from Lemma 2.2, (4.1), (4.6), and (4.8) that for $t \geq T_{1}, H(t) \geq E(\psi)$ and the equal sign holds if and only if for all $t \geq T_{1}, u$ is independent of $t$ and satisfies the equation $-\Delta u+f(x, u)=0$.

On the other hand, for $\theta$ being the constant appearing in Lemma 3.7, and for all $t \geq T_{1}$,

$$
-\frac{d}{d t}[H(t)-E(\psi)]^{\theta}=-\theta[H(t)-E(\psi)]^{\theta-1} \frac{d}{d t} H(t) .
$$

By Hölder's inequality, we get

$$
\begin{aligned}
& {[H(t)-E(\psi)]^{1-\theta} } \\
\leq \quad & C_{4}\left(|E(u)-E(\psi)|^{1-\theta}+\left\|u_{t}\right\|^{2(1-\theta)}+\left\|u_{t}\right\|_{L^{2}(\Gamma)}^{2(1-\theta)}+\|-\Delta u+f(x, u)\|^{2(1-\theta)}\right. \\
& \left.+\|-\Delta u+f(x, u)\|^{1-\theta}\left\|u_{t}\right\|^{1-\theta}+\left\|\nabla u_{t}\right\|^{2(1-\theta)}\right) .
\end{aligned}
$$

By Young's inequality we have

$$
\|-\Delta u+f(x, u)\|^{1-\theta}\left\|u_{t}\right\|^{1-\theta} \leq\|-\Delta u+f(x, u)\|+\left\|u_{t}\right\|^{(1-\theta) / \theta} .
$$

Thus

$$
\begin{aligned}
& {[H(t)-E(\psi)]^{1-\theta} } \\
\leq \quad & C_{4}\left(|E(u)-E(\psi)|^{1-\theta}+\left\|u_{t}\right\|^{2(1-\theta)}+\left\|u_{t}\right\|_{L^{2}(\Gamma)}^{2(1-\theta)}+\|-\Delta u+f(x, u)\|^{2(1-\theta)}\right. \\
& \left.+\|-\Delta u+f(x, u)\|+\left\|u_{t}\right\|^{(1-\theta) / \theta}+\left\|\nabla u_{t}\right\|^{2(1-\theta)}\right) .
\end{aligned}
$$

Since $(1-\theta) / \theta>1,2(1-\theta)>1$, it follows from Lemma 2.2 and (4.1) that for all $t \geq T_{1}$,

$$
[H(t)-E(\psi)]^{1-\theta} \leq C_{5}\left(|E(u)-E(\psi)|^{1-\theta}+\left\|u_{t}\right\|+\left\|u_{t}\right\|_{L^{2}(\Gamma)}+\|-\Delta u+f(x, u)\|+\left\|\nabla u_{t}\right\|\right) .
$$

We now consider all possibilities.

(1). If there is a $t_{0} \geq T_{1}$ such that at this time $H(t)=E(\psi)$, then for all $t>t_{0}$, as we have proved previously, $u$ is independent of $t$. Since $u\left(x, t_{n}\right) \rightarrow \psi$, then we are done.

(2). If there is $t_{0} \geq T_{1}$ such that for all $t \geq t_{0}, v=u-\psi$ satisfies the condition of Lemma 
3.7, i.e., $\|v\|_{H^{1}}<\widetilde{\beta}_{0}$, then for $\theta \in\left(0, \frac{1}{2}\right)$ appearing in Lemma 3.7, a combination of (4.6), (4.9) with (4.13), and application of Lemma 3.7 yields

$\frac{d}{d t}[H(t)-E(\psi)]^{\theta}+\frac{\widetilde{C}_{3} \theta}{C_{5}}\left(\left\|u_{t}\right\|+\|-\Delta u+f(x, u)\|+\left\|\nabla u_{t}\right\|+\left\|u_{t}\right\|_{L^{2}(\Gamma)}+\left\|u_{t}\right\|_{H^{\frac{1}{2}(\Gamma)}}\right) \leq 0$.

Integrating from $t_{0}$ to $t$, we get

$$
\begin{aligned}
& {[H(t)-E(\psi)]^{\theta} } \\
& +\frac{\widetilde{C}_{3} \theta}{C_{5}} \int_{t_{0}}^{t}\left(\left\|u_{t}\right\|+\|-\Delta u+f(x, u)\|+\left\|\nabla u_{t}\right\|+\left\|u_{t}\right\|_{L^{2}(\Gamma)}+\left\|u_{t}\right\|_{H^{\frac{1}{2}}(\Gamma)}\right) d \tau \\
\leq & {\left[H\left(t_{0}\right)-E(\psi)\right]^{\theta}<+\infty . }
\end{aligned}
$$

Since for $t \geq T_{1}, H(t)-E(\psi) \geq 0$, we have

$$
\int_{t_{0}}^{t}\left\|u_{t}\right\| d \tau<+\infty, \quad t \geq t_{0}
$$

This simply implies that as $t \rightarrow+\infty, u(t)$ converges in $L^{2}$. It follows from Lemma 2.2 that $u(t)$ is precompact in $H^{1}(\Omega)$. Hence, from the uniqueness of the limit we can deduce that

$$
\lim _{t \rightarrow \infty}\|u(t)-\psi\|_{H^{1}}=0 .
$$

(3). It follows from (4.1), (4.7) and Lemma 2.2 that for any $\sigma>0$ with $\sigma<\widetilde{\beta}_{0}$, there exists an integer $N$ such that when $n \geq N$,

$$
\left\|u\left(\cdot, t_{n}\right)-\psi\right\| \leq C\left\|u\left(\cdot, t_{n}\right)-\psi\right\|_{H^{1}}<\frac{\sigma}{2} .
$$

On the other hand, we deduce from the fact that $H(t)$ is decreasing in $\left[T_{1},+\infty\right)$ and it has a finite limit as $t \rightarrow+\infty$ that when $n \geq N$, for all $t \geq t_{n}$,

$$
\frac{C_{5}}{\widetilde{C}_{3} \theta}\left(\left(H\left(t_{n}\right)-E(\psi)\right)^{\theta}-(H(t)-E(\psi))^{\theta}\right)<\frac{\sigma}{2} \text {. }
$$

Define

$$
\bar{t}_{n}=\sup \left\{t>t_{n} \mid\|u(\cdot, s)-\psi\|_{H^{1}(\Omega)}<\widetilde{\beta}_{0}, \forall s \in\left[t_{n}, t\right]\right\} .
$$

It follows from (4.18) and continuity of the orbit in $H^{2}(\Omega)$ that $\bar{t}_{n}>t_{n}$ for all $n \geq N$. Then there are two possibilities:

(i). If there exists $n_{0} \geq N$ such that $\bar{t}_{n_{0}}=+\infty$, then from the previous discussions in (1), (2), we are done.

(ii) Otherwise, for all $n \geq N$, we have $t_{n}<\bar{t}_{n}<+\infty$, and for all $t \in\left[t_{n}, \bar{t}_{n}\right], E(\psi)<H(t)$. Then from (4.15) with $t_{0}$ being replaced by $t_{n}$, and $t$ being replaced by $\bar{t}_{n}$ we deduce that

$$
\int_{t_{n}}^{\bar{t}_{n}}\left\|u_{t}\right\| d \tau \leq \frac{C_{5}}{\widetilde{C}_{3} \theta}\left(\left(H\left(t_{n}\right)-E(\psi)\right)^{\theta}-\left(H\left(\bar{t}_{n}\right)-E(\psi)\right)^{\theta}\right)<\frac{\sigma}{2} .
$$

Thus, it follows that

$$
\left\|u\left(\bar{t}_{n}\right)-\psi\right\| \leq\left\|u\left(t_{n}\right)-\psi\right\|+\int_{t_{n}}^{\bar{t}_{n}}\left\|u_{t}\right\| d \tau<\sigma
$$


which implies that when $n \rightarrow+\infty$,

$$
u\left(\bar{t}_{n}\right) \rightarrow \psi \quad \text { in } L^{2}(\Omega)
$$

Since $u(t)$ is precompact in $H^{1}(\Omega)$, there exists a subsequence of $\left\{u\left(\bar{t}_{n}\right)\right\}$, still denoted by $\left\{u\left(\bar{t}_{n}\right)\right\}$, converging to $\psi$ in $H^{1}(\Omega)$. Then we can deduce that when $n$ is sufficiently large,

$$
\left\|u\left(\bar{t}_{n}\right)-\psi\right\|_{H^{1}(\Omega)}<\widetilde{\beta}_{0},
$$

which contradicts the definition of $\bar{t}_{n}$ that $\left\|u\left(\cdot, \bar{t}_{n}\right)-\psi\right\|_{H^{1}(\Omega)}=\widetilde{\beta}_{0}$. Thus, the theorem is proved.

Acknowledgment. We are grateful to professor M. Grasselli for calling our attention to the paper [10] for the proof of Lemma 2.2.

\section{REFERENCES}

[1] S. Aizicovici, E. Feireisl and F. Issard-Roch, Long-time convergence of solutions to a phasefield system, Mathematical Methods in the Applied Sciences, 24, (2001), 277-287. MR1818896 (2002b:35081)

[2] A. V. Babin and M. I. Vishik, Attractors of Evolution Equations, North-Holland, Amsterdam, 1992. MR.1156492 (93d:58090)

[3] J. Ball, Global attractors for damped semilinear wave equations, Discrete and Continuous Dynamical Systems, Vol. 10, No. 1\&2(2004), 31-52. MR2026182(2005a:37149)

[4] V. Barbu, Nonlinear Semigroups and Differential Equations in Banach Spaces, Noordhoff, 1976. MR 0390843(52:11666)

[5] H. Brézis, Opérateurs Maximaux Monotones et semi-groupes de contractions dans les espaces de Hilbert, North-Holland, Amsterdam, 1973. MR0348562 (50:1060)

[6] R. Chill, On the Lojasiewicz-Simon gradient inequality, J. Funct. Anal., Vol. 201(2003), 572-601. MR $1986700(2005 \mathrm{c}: 26019)$

[7] I. Chueshov, M. Eller and I. Lasiecka, On the attractor for a semilinear wave equation with critical exponent and nonlinear boundary dissipation, Comm. PDE. Vol. 27(2002), 1901-1951. MR1941662 (2003m:35034)

[8] E. Feireisl, Global attractors for semilinear damped wave equations with supercritical exponent, Journal of Differential Equations, Vol. 116 (1995), 431-447. MR1318582 (96a:35120)

[9] E. Feireisl, F. Issard-Roch, and H. Petzeltova, Long-time behaviour and convergence towards equilibria for a conserved phase field model, Discrete and Continuous Dynamical Systems, Vol. 10, No. 1\&2(2004), 239-252. MR 2026193 (2004m:35047)

[10] M. Grasselli, V. Pata, Asymptotic behavior of a parabolic-hyperbolic system, Commun. Pure Appl. Anal. 3(2004), 849-881. MR.2106302 (2005h:35150)

[11] J.K. Hale, Asymptotic Behavior of Dissipative Systems, AMS Math. Surveys and Monographs, 25, Providence, Rhode Island, 1988. MR0941371 (89g:58059)

[12] A. Haraux, Semilinear hyperbolic problems in bounded domains, Mathematical Reports, Vol. 3, Harwood Gordon Breach, New York, 1987. MR.1078761 (91m:35150)

[13] A. Haraux and M.A. Jendoubi, Convergence of bounded weak solutions of the wave equation with dissipation and analytic nonlinearity, Calc. Var. vol. 9 (1999), 95-124. MR1714129 (2000h:35110)

[14] S.Z. Huang and P. Takáč, Convergence in gradient-like systems which are asymptotically autonomous and analytic, Nonlinear Analysis, 46 (2001), 675-698. MR1857152 (2002f:35125)

[15] M.A. Jendoubi, A simple unified approach to some convergence theorems of L. Simon, J. Funct. Anal., 153 (1998), 187-202. MR1609269 (99c:35101)

[16] M.A. Jendoubi, Convergence of global and bounded solutions of the wave equation with linear dissipation and analytic nonlinearity, Journal of Differential Equations, 144 (1998), 302-312. MR 1616964 (99e:35149)

[17] I. Lasiecka, Mathematical Control Theory of Coupled PDE's, CBMS-NFS Lecture Notes. SIAM, Philadelphia, 2002. MR1879543 (2003a:93002) 
[18] I. Lasiecka, D. Tataru, Uniform boundary stabilization of a semilinear wave equation with nonlinear boundary damping, Differential and Integral Equations, vol. 6 (1993), 507-533. MR1202555 (94c:35129)

[19] W. Li, Long-time convergence of solution to phase-field system with Neumann boundary conditions, to appear in Chinese Annals of Mathematics A.

[20] S. Lojasiewicz, Une propriété topologique des sous-ensembles analytiques réels. Colloques Internationaux du C.N.R.S. \#117, (1963), 87-89. MR0160856 (28:4066)

[21] S. Lojasiewicz, Sur la géométrie semi- et sous-analytique. Ann. Inst. Fourier (Grenoble) 43, (1963), 1575-1595. MR1275210 (96c:32007)

[22] S. Lojasiewicz, Ensemble semi-analytique. Bures-sur-Yvette: IHES (1965).

[23] J. Lions and E. Magenes, Nonhomogeneous Boundary Value Problems, Springer, Berlin, 1973.

[24] H. Matano, Convergence of solutions of one-dimensional semilinear parabolic equations, J. Math. Kyoto Univ. 18-2 (1978), 221-227. MR0501842 (80a:35016)

[25] L. Nirenberg, Topics in Nonlinear Functional Analysis, Courant Institute of Mathematical Science, New York, 1974. MR0488102 (58:7672)

[26] P. Polačik and K.P. Rybakowski, Nonconvergent bounded trajectories in semilinear heat equations, J. Diff. Eqs., 124 (1996), 472-494. MR.1370152 (96m:35176)

[27] P. Polačik and F. Simondon, Nonconvergent bounded solutions of semilinear heat equations on arbitrary domains, J. Diff. Eqs., 186 (2002), 586-610. MR.1942223 (2003h:35115)

[28] P. Rybka and K.H. Hoffmann, Convergence of solutions to Cahn-Hilliard equation, Comm. PDEs, 24 (5\&6), (1999), 1055-1077. MR1680877 (2001a:35028)

[29] R. Showalter, Monotone Operators in Banach Spaces and Nonlinear Partial Differential Equations, AMS, Providence, 1997. MR:1422252 (98c:47076)

[30] G. Sell and Y. You, Dynamics of Evolutionary Equations, Springer, New York, 2002. MR1873467 (2003f:37001b)

[31] L. Simon, Asymptotics for a class of nonlinear evolution equations, with applications to geometric problems, Ann. of Math., 118 (1983), 525-571. MR0727703 (85b:58121)

[32] R. Temam, Infinite-dimensional Dynamical Systems in Mechanics and Physics, Appl. Math. Sci., 68, Springer-Verlag, New York, 1988. MR0953967 (89m:58056)

[33] G.F. Webb, Compactness of bounded trajectories of dynamical systems in infinite-dimensional spaces, Proc. Roy. Soc. Edinburgh, 84 A(1979), 19-34. MR.5549869 (80j:34084)

[34] Hao Wu and Songmu Zheng, Convergence to equibrium for the Cahn-Hilliard equation with dynamic boundary conditions, Journal of Differential Equations, Vol. 204, (2004), 511-531. MR2085545 (2005i:35158)

[35] T.I. Zelenyak, Stabilization of solutions of boundary value problems for a second-order parabolic equation with one space variable, Differentsial'nye Uravneniya, (1968), 17-22.

[36] Z. Zhang, Asymptotic behavior of solutions to the phase-field equations with Neumann boundary conditions, Comm. Pure Appl. Anal. 4, (2005), 683-693.

[37] Songmu Zheng, Nonlinear Evolution Equations, Pitman Monographs and Surveys in Pure and Applied Mathematics, 133, Chapman \& Hall/CRC, Boca Raton, Florida, 2004. MR2088362

[38] Songmu Zheng and M. Chipot, Asymptotic behavior of solutions to nonlinear parabolic equations with nonlocal terms, Asymptotic Analysis, 45 (3,4) (2005), 301-312. 